Global Sustainability

cambridge.org/sus

\section{Editorial}

Cite this article: Reynolds JL (2018). The politics and governance of negative emissions technologies. Global Sustainability 1, e12, 1-3. https://doi.org/10.1017/sus.2018.13

Received: 5 September 2018

Revised: 22 October 2018

Accepted: 7 November 2018

Author for correspondence:

J. L. Reynolds, E-mail: reynolds@law.ucla.edu

(C) The Author(s) 2018. This is an Open Access article, distributed under the terms of the Creative Commons AttributionNonCommercial-ShareAlike licence (http:// creativecommons.org/licenses/by-nc-sa/4.0/), which permits non-commercial re-use, distribution, and reproduction in any medium, provided the same Creative Commons licence is included and the original work is properly cited. The written permission of Cambridge University Press must be obtained for commercial re-use.

\section{CAMBRIDGE} UNIVERSITY PRESS

\title{
The politics and governance of negative emissions technologies
}

Jesse L. Reynolds ${ }^{1,2}$

${ }^{1}$ University of California, Los Angeles, School of Law, Emmett Institute on Climate Change and the Environment, 385 Charles E. Young Drive East, 1242 Law Building, Los Angeles, California 90095, USA and ${ }^{2}$ Utrecht University, School of Law, Utrecht Centre for Water, Oceans and Sustainability Law, Newtonlaan 201, 3584 BH Utrecht, The Netherlands

It is well known that the Paris Agreement aims to keep climate change - perhaps the greatest current challenge to global sustainability - to within $2{ }^{\circ} \mathrm{C}$ of warming, and to strive for $1.5^{\circ} \mathrm{C}$. Many people point toward the scenarios used by the Intergovernmental Panel on Climate Change (IPCC) and conclude that this is feasible if only our leaders had the political will to do what's necessary. However, it is less widely known that these scenarios assume the use of technologies to remove carbon dioxide from the atmosphere at very large scales. As global emissions have not lessened in the seven years since the scenarios' development, more recent projections to stay within $2{ }^{\circ} \mathrm{C}$ and especially $1.5^{\circ} \mathrm{C}$ rely upon both more aggressive emissions cuts and larger scales of 'negative emission technologies' (NETs). Yet not only are NETs' actual development and scalability still uncertain, the technologies would present social and environmental risks of their own.

In some ways, negative emissions are not qualitatively new. The 1992 UN Framework Convention on Climate Change (UNFCCC) speaks repeatedly of the enhancement of sinks and reservoirs in order to remove greenhouse gases from the atmosphere. The 2015 Paris Agreement more overtly calls for a "balance between anthropogenic emissions by sources and removals by sinks of greenhouse gases," that is, net zero emissions.

Yet in other, important ways, NETs are novel. The drafters of the UNFCCC and its later Kyoto Protocol had in mind land-use change and forestry activities, such afforestation and reforestation. By contrast, several negative emissions technologies have become objects of serious study during the last decade. First, carbon dioxide could be directly captured from the air and stored, perhaps underground. Second, plants could be grown, pulling carbon dioxide from the air. They could later be burnt, producing bioenergy while the released carbon dioxide could be captured and stored. Third, the natural weathering of minerals - a process that indirectly uses atmospheric carbon dioxide - could be accelerated. Finally, the oceans could be fertilized, accelerating the so-called 'biological pump' that naturally sequesters carbon in deep waters. Together, these proposed techniques could help lower net emissions, and unlike emissions abatement, they could actually reduce atmospheric carbon dioxide concentrations following an 'overshoot' of a concentration target.

At the same time, NETs are also problematic in multiple ways. At sufficient scales, they each pose physical and environmental risks. Captured carbon dioxide from direct air capture or from 'bioenergy with carbon capture and storage' (BECCS) could leak. Enhanced weathering would use outdoor industrial processes akin to mining. NETs would also present social challenges. BECCS would need large amounts of arable land, which would compete with agriculture and drive up food prices. In other words, NETs might be able to contribute to sustainable development, yet some of their secondary effects seem to threaten the Sustainable Development Goals.

Many observers wonder whether the growing interest in NETs is itself a risk. As described above, widely-used, model-based scenarios of limited global warming rely on NETs at very large scales. To give a sense of this, the 15 tons of carbon dioxide per year that we might need to remove by the end of the century is approximately six times the mass of current annual global cereals production. This would constitute a genuine societal transformation, but it is unclear whether humanity will be able to undertake an intentional physical task of this magnitude, or whether NETs are merely a device to transform the impossible into the seemingly attainable. Reliance on future yet uncertain large-scale NETs, especially to later compensate for excessive present and near-term emissions, could weaken emissions abatement efforts.

And deeper questions linger. In some ways, scaling up NETs would represent an acknowledgement that humans have assumed the responsibility to manage the global carbon cycle. This, in turn, could contribute to the realization of the Anthropocene, in which we are a dominant force affecting natural systems.

Despite the growing realization of NETs' necessity, their international politics and policies remain amorphous and emerging. Clearly, there will be politics and governance of NETs. Who 
stands to gain from their research, development, and use, and who might lose out? Who would have a voice in decision-making, and who might not? Can the traditional tenets of environmentalism and tools of environmental policy - which have been oriented toward reducing humans' impacts on the natural world - manage NETs? If NETs imply a societal transformation, how can such a transformation be responsibly accelerated. Simultaneously, there will be governance by NETs. That is, the development of NETs may influence and perhaps catalyse other responses to climate change. These matters will be contested; how they are contested and by whom may be novel and surprising.

Because these and related questions remain underexplored, I organized a workshop that brought together 25 international experts to discuss the politics and governance of NETs. ${ }^{i}$ It took place in the historic city centre of Utrecht, The Netherlands, and was part of the 'Innovations in Climate Governance' (INOGOV) COST Action. The workshop's objective was to bring together diverse scholars in the social sciences to deepen the understandings of the challenges and opportunities of NETs' research, development, and possible implementation. Over two days, participants presented and commented on papers on law, policy, domestic and international politics, the sociology of knowledge and expertise, ethics, and public perception. Common themes across the papers included: To what extent is it useful to (dis)aggregate NETs and related technologies? How can both elite expertise and stakeholders' perspectives be appropriately integrated into decision-making? What are the roles of existing governance mechanisms and institutions, and are new ones needed? What relevant lessons can be drawn from other emerging technologies?

Social geographer Holly Jean Buck uses a specific analogous case to draw lessons. Some residents of the rural Imperial Valley in the southern California desert increasingly envision it as a future site of large-scale renewable energy. She draws from visits there and semi-structured interviews to understand how local actors conceptualize and contest climate change technologies, including carbon negative ones. Buck concludes that entrenched interests could play important roles in NETs' development, that concerns are not merely resistance to change, and that policies and incentives should be adapted to local conditions.

Like most environmental legal regimes, US law regulates what substances may be introduced into the environment. Yet NETs especially direct air capture - would remove pollutants from the atmosphere. Tracy Hester of the University of Houston considers whether this legal gap could present governance challenges. For example, assuming that a direct air capture operator would own the resulting carbon dioxide, has it converted a formerly public resource into a private one? Hester suggests that environmental law would better focus on impacts on ecological systems instead of on the release of pollutants.

Two papers considered how climate experts produce knowledge and frame questions regarding NETs. In the first of these, Kate Dooley, Peter Christoff, and Kimberly Nicholas of the University of Melbourne and Lund University explored the co-production of model-based knowledge and policy demands. By examining the role of BECCS and afforestation in scenarios generated by multiple integrated assessment models under four different Shared Socio-economic Pathways, they find that the scales of land-based negative emissions would likely force difficult trade-offs with other goals related to sustainable development. Dooley and her colleagues conclude that the perception of the outputs as 'objective knowledge' legitimizes NETs as a response option without any critical interrogation of the inherent normative choices.

In the second paper on knowledge production and framing, Silke Beck and Martin Mahony consider the history of the IPCC, which plays an essential role at the science-policy interface. Drawing from science and technology studies, they suggest that by encouraging the reliance on BECCS in models, the IPCC has shifted from assessing existing scientific evidence towards projecting policy solutions. Beck and Mahony warn that, through its creation of politically powerful futures, the IPCC exercises a 'world-making' power. Ultimately, this challenges the notion that such assessment bodies can be objective and suggests that political contexts and implications should be considered.

Lancaster University's Nils Markusson, Duncan McLaren, and David Tyfield identify three approaches that could be used to investigate whether and to what extent the consideration of NETs might deter or delay emissions abatement. The 'realist register' is individualist, managerialist and economist, and has dominated the discourse thus far. They argue for moving to the cultural register, which recognizes scientists as epistemological power brokers, and onward to a cultural political economy register, which considers social and economic relations. This would reveal how NETs are not a substitute for emissions abatement yet might crowd it out.

Decision-making with regards to a proposed endeavour on the scale of NETs, with numerous diverse expected impacts, could and should be guided by ethics. In his article in this collection, Dominic Lenzi offers one of the first overviews of the ethics of NETs. He identifies three core concerns: that NETs might undermine emissions abatement; that they might encourage a dangerous gamble in terms of climate policy; and that they might fail to deliver the expected carbon removal. Using three possible futures, Lenzi concludes that these concerns' severity will depend upon the precise conditions of the NETs' implementation.

Clair Gough and her colleagues at the Tyndall Centre for Climate Change Research identify six core challenges to the governance of BECCS. First, in order to make a significant contribution to staying within carbon budgets, BECCS depends on ambitious emissions abatement in the near-term. Second, because its supply chain would use materials and energy, net (negative) emissions must be accurately quantified across its full lifecycle. The third issue is whether BECCS could deliver at sufficient scales. After all, current assumed scales imply an additional 300-600 Mha land dedicated to biomass production. Fourth, could this be done sustainably? Fifth, negative emissions from BECCS would need to be integrated into broader climate policy. Finally, how could BECCS fit with international climate agreements, particularly regarding the allocation of its costs and benefits?

The relevant issues are complex. NETs will function across a wide range of temporal and spatial scales, affect many interrelated natural and social systems, and involve multiple sectors of society, government, and the economy. Understanding their politics and governance requires not only a diversity of perspectives, but their integration into interdisciplinary conversations and research. Such an approach was among the workshop's goals, and is why seven of its papers are collected in this debut issue of Global Sustainability. I look forward to how this admirably interdisciplinary journal explores coupled social-ecological systems in the upcoming issues.

Financial support. This work was supported by the COST Action IS1309 Innovations in Climate Governance: Sources, Patterns and Effects (INOGOV). 


\section{Conflict of interest. None.}

\section{Note}

i I thank Global Sustainability for providing an outlet for this collection of articles and for publishing them with Open Access, as well as its editorial staff for their assistance. The workshop would not have been possible without the support of the INOGOV COST Action and the Utrecht Centre for Water, Oceans, and Sustainability Law of Utrecht University. Most importantly, I am grateful for the contributions of the authors and other workshop participants, who have all helped advance an important conversation. 